\title{
Exclusion of PITX2 Mutations as a Major Cause of CHARGE Association
}

\author{
Donna M. Martin, ${ }^{1,2} *$ Frank J. Probst, ${ }^{2}$ Sharon E. Fox, ${ }^{2}$ Lisa A. Schimmenti, ${ }^{3}$ Elena V. Semina, ${ }^{4}$ \\ Margaret A. Hefner, ${ }^{5}$ John W. Belmont, ${ }^{6}$ and Sally A. Camper ${ }^{2}$ \\ ${ }^{1}$ Department of Pediatrics, The University of Michigan Medical School, Ann Arbor, Michigan \\ ${ }^{2}$ Department of Human Genetics, The University of Michigan Medical School, Ann Arbor, Michigan \\ ${ }^{3}$ Department of Human Genetics, Pediatrics and the Mental Retardation Research Center, University of California, \\ Los Angeles \\ ${ }^{4}$ Department of Pediatrics, University of Iowa, Iowa City, Iowa \\ ${ }^{5}$ Department of Pediatrics, St. Louis University School of Medicine, St. Louis, Missouri \\ ${ }^{6}$ Department of Pediatrics, Baylor College of Medicine, Houston Texas
}

\begin{abstract}
CHARGE is a nonrandom association of ocular coloboma, congenital heart defects, atresia of the choanae, retarded growth and development, genital hypoplasia, and ear anomalies including deafness. The cause of CHARGE remains unknown; however, there is considerable evidence of an underlying genetic basis, as discussed by Tellier et al. [1996: Clin Genet 50:548-550; 1998: Am J Med Genet 76:402-409] and by Martin et al. [2001: Am J Med Genet 99:115-119]. Based on the ocular, cardiac, and craniofacial expression pattern of Pitx2, a homeodomain transcription factor, and the pleiotropic effects of loss of PITX2 function in both mouse and human, we hypothesized that PITX2 mutations may contribute to the multiple phenotypic anomalies present in CHARGE individuals. By direct sequencing of DNA from 29 individuals with CHARGE, we did not identify any mutations in PITX2. We did, however, identify two PITX2 sequence polymorphisms. Large deletions of PITX2 were excluded in most patients by heterozygosity in at least one of several polymorphic markers near the PITX2 locus. Together, these data indi-
\end{abstract}

Grant sponsor: NIH; Grant numbers: NICHH K08 HD 40288, ROI HD34283; The University of Michigan Sequencing Core; Grant numbers: P30 AR20557, P30CA 46592, P30 DK20572.

Frank J. Probst's present address is Department of Pediatrics, Cornell Medical Center, New York, NY.

*Correspondence to: Donna M. Martin, Department of Pediatrics, Genetics Division, The University of Michigan, 35204 Medical Science Research Building I, University of Michigan Medical School, Ann Arbor, MI 48109-0688.

E-mail: donnamm@umich.edu

Received 21 January 2002; Accepted 12 February 2002

DOI 10.1002/ajmg.10473 cate that PITX2 mutations are unlikely to be a major contributing cause of the multiple anomalies present in individuals with CHARGE. (c) 2002 Wiley-Liss, Inc.

KEY WORDS: CHARGE; PITX2; mutation; sequence polymorphism

\section{INTRODUCTION}

CHARGE association was first described in 1979 [Hall, 1979], and the CHARGE acronym was proposed in 1981 [Pagon et al., 1981]. CHARGE is a collection of congenital anomalies including ocular coloboma (anterior or posterior), cardiac defects, choanal atresia (bony and/or membranous), delayed growth and development, external and inner ear anomalies, deafness, and cranial nerve dysfunction. Major diagnostic features of CHARGE syndrome, are present-a potential subset of individuals affected by CHARGE, and include coloboma, characteristic ear and cranial nerve abnormalities, and choanal atresia [Blake et al., 1998]. CHARGE affects the craniofacial region, teeth, and cardiac outflow tract, leading to the suggestion that CHARGE is a disorder of neural crest migration and development [Bolande, 1997].

Most case of CHARGE are sporadic with an unidentifiable cause. However, there is considerable evidence that CHARGE may have a genetic basis, including an association with increased paternal age [Tellier et al., 1996], rare familial cases, concordance among monozygotic twins, and discordance among dizygotic twins [Tellier et al., 1998]. Several chromosomal rearrangements have been reported among CHARGE individuals, including duplications of $14 \mathrm{q}$ and $1 \mathrm{q}$ [Dev et al., 1985; North et al., 1995] and a balanced translocation involving 2p14 and 7q21 [Martin et al., 2001]. To date, no single chromosomal region appears to be preferentially involved in the CHARGE phenotype. CHARGE might result from mutations in a dosage-sensitive 
developmental pathway required by all the involved tissues. Alternatively, pleiotropic activity of a single factor in multiple developmental fields could explain the complex phenotype. The rarity of familial CHARGE cases, combined with high early mortality among CHARGE individuals, renders positional cloning and linkage analysis difficult. Instead, candidate gene analysis is a potentially useful approach to understanding the etiology of multiple congenital anomaly conditions, and has been used successfully to exclude PAX2 mutations as a major cause of CHARGE [Tellier et al., 2000].

Pitx2, a transcription factor essential for proper formation of the eyes, heart, and craniofacial region, is expressed in several areas of the developing murine neural crest [Hjalt et al., 2000]. In humans, PITX2 mutations cause ocular anomalies, including AxenfeldRieger syndrome (ARS), a haploinsufficiency condition affecting the eyes, teeth and umbilicus with associated abnormalities of the craniofacial region (midfacial hypoplasia, prognathism) [Semina et al., 1996; Alward et al., 1998; Doward et al., 1999; Kozlowski and Walter, 2000; Perveen et al., 2000; Priston et al., 2001]. Some patients with ARS and unknown mutation also exhibit congenital heart disease (atrial septal defect, conotruncal anomalies), choanal atresia, and growth hormone deficiency, indicating some clinical overlap with CHARGE association [Feingold et al., 1969; Sadeghi-
Nejad and Senior, 1974; Brooks et al., 1989]. In mice, complete loss of Pitx2 results in embryonic lethality, with severe ocular, cardiac, and abdominal abnormalities, and disruptions in tooth, pituitary and craniofacial development [Gage et al., 1999; Lin et al., 1999]. The PITX2 locus produces three isoforms designated PITX2a, $b$, and $c$ that arise through differential splicing and alternative promoter usage (Fig. 1) [Arakawa et al., 1998]. In mice, the Pitx2c isoform plays a particular role in early mesoderm differentiation, establishment of embryonic left-right axis, and cardiac development [Liu et al., 2001]. The striking overlap between the phenotypic anomalies in Pitx2-deficient mice and the clinical features of CHARGE led us to hypothesize that PITX2 mutations may contribute to the multiple anomalies present in individuals with CHARGE.

We found, by direct sequencing of genomic DNA from 29 individuals with CHARGE, no identifiable mutations in the coding region or intron-exon borders of PITX2. Two polymorphic variants in the PITX2 coding sequence were identified based on comparative sequencing of unaffected parent DNA samples. Large deletions near the PITX2 locus were also excluded in 28 of 29 CHARGE individuals by heterozygosity at highly polymorphic markers near PITX2. Taken together, these results indicate that PITX2 mutations are unlikely to be a major cause of the multiple congenital anomalies seen in CHARGE individuals.
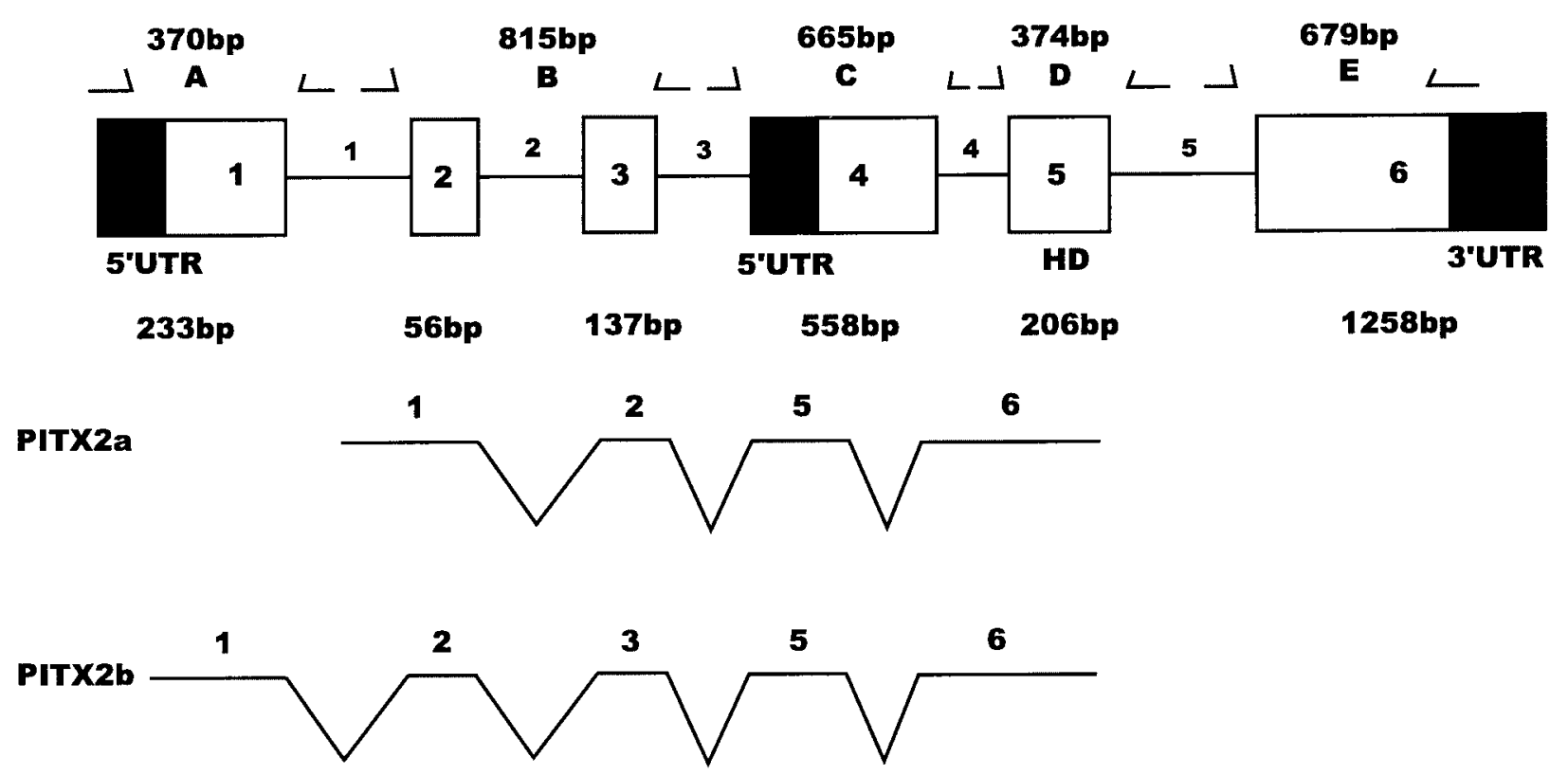

4

5

6

PITX2c

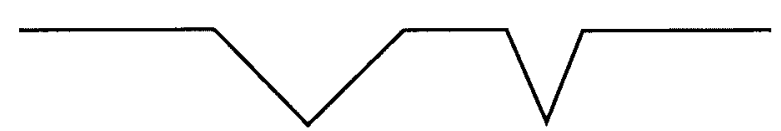

Fig. 1. Diagram of human PITX2 gene and three different mRNA isoforms (PITX2a, PITX2b, PITX2c), with exons numbered in boxes and introns numbered above intervening segments. Sizes of exons in base pairs are given below each box; filled areas are untranslated regions. Introns are not drawn to scale. Primers used for sequencing are indicated as arrows, with five polymerase chain reaction products (A-E) and sizes in base pairs indicated. Adapted from Amendt et al., 2000. 


\section{METHODS \\ Patient Information}

Patient and parent samples (10 $\mathrm{mL}$ peripheral blood) were obtained with IRB consent from each of three participating institutions (University of Michigan, Baylor College of Medicine, University of California, Los Angeles). Twenty-seven of 29 patients were from the CHARGE Syndrome Foundation Research Resource. Inclusion criteria were a clinical diagnosis of CHARGE on the basis of at least four of the originally described six major features (coloboma, heart defects, atresia of the choanae, retarded growth and development, genital hypoplasia, and ear anomalies including deafness). Clinical features of the patients are outlined in Table I. All but two patients had normal peripheral blood chromosomes and no 22q11 deletion by fluorescence in situ hybridization testing or STR genotyping; similar testing information was not available for the other two patients. Prior approval for this study was obtained through the Internal Review Board of the University of Michigan.

\section{DNA Isolation and Sequencing}

Peripheral blood samples were obtained from affected patients and their parents. Lymphoblastoid cell lines were prepared from blood samples and DNA isolated with QIAGEN columns [Qiagen, Valencia, $\mathrm{CA}$ ]. Primer pairs were as shown in Figure 1: region A, AATCTCTGCTGACGTCACGT and CCAGACTCGCATTATCTCAC; region $\mathrm{B}$, TAGTCTCATCTGAGCC-

TABLE I. Clinical Features of CHARGE Subjects $(\mathrm{N}=29)$

\begin{tabular}{lcc}
\hline Feature & $\begin{array}{c}\text { No. of } \\
\text { individuals }\end{array}$ & $\begin{array}{c}\text { Ratio or } \\
\text { percentage }\end{array}$ \\
\hline Male: female & $14: 15$ & $1: 1.07$ \\
Ocular coloboma & 26 & $90 \%$ \\
Heart defect & 25 & $86 \%$ \\
Undescended testes or & 12 & $86 \%$ \\
$\quad$ small penis & & \\
Ear malformation & 24 & $83 \%$ \\
Hearing loss & 24 & $83 \%$ \\
Development delay & 20 & $72 \%$ \\
Swallowing problems & 19 & $65 \%$ \\
Choanal atresia or stenosis & 17 & $59 \%$ \\
Respiratory disease & 17 & $59 \%$ \\
Recurrent otitis media & 17 & $59 \%$ \\
Growth retardation & 15 & $52 \%$ \\
Facial nerve palsy & 14 & $48 \%$ \\
Increased secretions & 12 & $41 \%$ \\
Renal or other urinary & 11 & $38 \%$ \\
$\quad$ tract anomaly & & $24 \%$ \\
Hernia (unspecified) & 7 & $21 \%$ \\
Cleft lip or palate & 6 & $21 \%$ \\
Tracheoesophageal fistula & 6 & $17 \%$ \\
CNS defect & 5 & $10 \%$ \\
Growth hormone deficiency & 3 & \\
\hline Percentage given is that of & & \\
\hline
\end{tabular}

${ }^{\text {a }}$ Percentage given is that of the total number of males. Other data are presented as percentage of the total $(\mathrm{N}=29)$. Single cases with microcephaly, seizures, absence of thymus, hypoparathyroidism were also reported. Average age at evaluation was 5.7 years. Ocular coloboma is defined as involving the retina and/or iris. Hearing loss includes sensorineural or conductive types, and respiratory disease includes multiple pneumonias or breathing problems.
CTGC and TTCTTGCGCTTTCGCCCGA; region C, CTTGACACTTCTCTGTCAGG and AAGCGGGAATGTCTGCAGG; region D, CAGCTCTTCCACGGCTTCT and TTCTCTCCTGGTCTACTTGG; region E, GTAATCTGCACTGTGGCATC and AGTCTTTCAAGGGCGGAGTT. PCR was at $55^{\circ} \mathrm{C}$ annealing temperature. Amplified products were separated on agarose gels, reamplified, separated on agarose gels, purified with QIAEX II or QIAQUICK kits (Qiagen) and sequenced directly, using Applied Biosystems DNA Sequencers with the manufacturer's protocols (BigDye Version1 terminator chemistry, DNA Sequencing core, University of Michigan). Chromatograms were visualized with Sequencer software (Gene Codes Corporation, Ann Arbor, Michigan) and compared to the published genomic PITX2 sequence [Genbank accession number AF238048, or chr4:118538103-118558100 in the April 1, 2001 freeze of the Golden Path sequence assembly (http://genome.ucsc.edu/)].

\section{RESULTS AND DISCUSSION}

In this study, we demonstrate absence of PITX2 mutations in a series of 29 individuals with CHARGE association. We sequenced the PITX2 coding region and intron-exon boundaries, and identified two sequence changes in introns, both near splice junctions but not within consensus splice sites (Table II). These sequence changes were also present in unaffected parents, and likely represent polymorphic variants. Heterozygosity was detected in 28 of 29 affected patients for at least one of three polymorphic markers (D4S406, D4S2989, D4S2945 with heterozygosity values of $0.8843,0.8423$ and 0.783 , respectively) near the PITX2 locus. Data presented in this study do not exclude mutations in the distal 3' UTR or in complete intronic regions of PITX2, nor have we excluded mutations in the PITX2 promoter region that may affect transcript levels. Nevertheless, our observations indicate that mutations in PITX2 are unlikely to contribute to the phenotypic abnormalities present in CHARGE association.

PITX2 mutations have been identified in association with a variety of clinical features, including Peters' anomaly, iridogoniodysgenesis, iris hypoplasia, and ARS; many of these patients also have maxillary hypoplasia and prognathism [Semina et al., 1996; Alward et al., 1998; Amendt et al., 1998; Kulak et al., 1998; Doward et al., 1999; Amendt et al., 2000; Kozlowski and Walter, 2000; Perveen et al., 2000; Priston et al., 2001; Saadi et al., 2001]. In addition to the phenotypic variability, there is considerable genetic heterogeneity among patients with ARS, and in a recent study of 76 individuals, eight were found to have PITX2 mutations [Perveen et al., 2000]. Several different PITX2 mutations have been reported, including

TABLE II. PITX2 Sequence Variants

\begin{tabular}{lc}
\hline Sequence location & Sequence \\
\hline Intron 2 & CCCTCTTTCT (A/C) CTCCGGCCT \\
Intron 3 & GCGTGGGGGGGG (G) CGGGCAG
\end{tabular}


nonsense mutations resulting in prematurely truncated PITX2 protein, missense mutations with decreased PITX2 homeodomain DNA binding, and a recently identified dominant negative mutation that leads to overexpression of a novel PITX2 protein [Priston et al., 2001].

The ocular abnormalities in patients with ARS and PITX2 mutations typically involve the anterior segment of the eye, including the iris and lens. Similar anterior segment abnormalities (corectopia) are present in mice with complete loss of Pitx2, consistent with Pitx 2 mRNA and protein expression in the lens and anterior ocular mesenchyme during development [Gage et al., 1999; Hjalt et al., 2000]. Surprisingly, however, loss of Pitx 2 function in mice also leads to absence of the extraocular muscles and atresia of the optic nerve, even though only the extraocular muscles (and not the optic nerve) express Pitx2 [Gage et al., 1999]. Thus, loss of Pitx2 in the ocular mesenchyme may negatively influence development of adjacent posterior structures like the optic nerve and extraocular muscles. Data presented here indicate the PITX2 mutations are not a major contributing cause of the ocular, cardiac, and craniofacial anomalies in CHARGE association; however, further studies will determine whether genes that are regulated by PITX2 are involved in the CHARGE phenotype.

\section{ACKNOWLEDGMENTS}

We thank Jeff Murray for helpful discussions and critically reviewing the manuscript. The CHARGE Syndrome Foundation Research Resource was developed in the Tissue Culture Core Laboratory of the BCM Mental Retardation Research Center (L. Molinari Supervisor) NIH HD24064. The CHARGE Syndrome Foundation (www.CHARGEsyndrome.org) kindly provided clinical information, and their continued support is appreciated. This study was supported in part by NICHD KO8 HD 40288 (D.M.M.), RO1 HD34283 (S.A.C.), UCLA Pediatrics, Child Health Research Center Award P30 HD34650, Research to Prevent Blindness and the UCLA Council on Research (L.A.S.), RO1 HD39056 (J.W.B.).

\section{REFERENCES}

Alward WL, Semina EV, Kalenak JW, Heon E, Sheth BP, Stone EM, Murray JC. 1998. Autosomal dominant iris hypoplasia is caused by a mutation in the Rieger syndrome (RIEG/PITX2) gene. Am J Ophthalmol 125:98-100.

Amendt BA, Sutherland LB, Semina EV, Russo AF. 1998. The molecular basis of Rieger syndrome. Analysis of Pitx2 homeodomain protein activities. J Biol Chem 273:20066-20072.

Amendt BA, Semina EV, Alward WL. 2000. Rieger syndrome: a clinical, molecular, and biochemical analysis. Cell Mol Life Sci 57:1652-1666.

Arakawa H, Nakamura T, Zhadanov AB, Fidanza V, Yano T, Bullrich F, Shimizu M, Blechman J, Mazo A, Canaani E, Croce CM. 1998. Identification and characterization of the ARP1 gene, a target for the human acute leukemia ALL1 gene. Proc Natl Acad Sci USA 95:4573-4578.

Blake KD, Davenport SL, Hall BD, Hefner MA, Pagon RA, Williams MS, Lin AE, Graham JM Jr. 1998. CHARGE association: an update and review for the primary pediatrician. Clin Pediatr 37:159-173.

Bolande RP. 1997. Neurocristopathy: its growth and development in 20 years. Pediatr Pathol Lab Med 17:1-25.

Brooks JK, Coccaro PJ Jr, Zarbin MA. 1989. The Rieger anomaly concomitant with multiple dental, craniofacial, and somatic midline anomalies and short stature. Oral Surg Oral Med Oral Pathol 68:717724.

Dev V, Butler M, Philips J. 1985. 1q duplication due to unequals crossover in a patient with CHARGE association and DiGeorge sequence. Am J Hum Genet 37:90A.

Doward W, Perveen R, Lloyd IC, Ridgway AE, Wilson L, Black GC. 1999. A mutation in the RIEG1 gene associated with Peters' anomaly. J Med Genet 36:152-155.

Feingold M, Shiere F, Fogels HR, Donaldson D. 1969. Rieger's syndrome. Pediatrics 44:564-569.

Gage PJ, Suh H, Camper SA. 1999. Dosage requirement of Pitx2 for development of multiple organs. Development 126:4643-4651.

Hall BD. 1979. Choanal atresia and associated multiple anomalies. J Pediatr 95:395-398.

Hjalt TA, Semina EV, Amendt BA, Murray JC. 2000. The Pitx2 protein in mouse development. Dev Dyn 218:195-200.

Kozlowski K, Walter MA. 2000. Variation in residual PITX2 activity underlies the phenotypic spectrum of anterior segment developmental disorders. Hum Mol Genet 9:2131-2139.

Kulak SC, Kozlowski K, Semina EV, Pearce WG, Walter MA. 1998. Mutation in the RIEG1 gene in patients with iridogoniodysgenesis syndrome. Hum Mol Genet 7:1113-1117.

Lin CR, Kioussi C, O’Connell S, Briata P, Szeto D, Liu F, Izpisua-Belmonte JC, Rosenfeld MG. 1999. Pitx2 regulates lung asymmetry, cardiac positioning and pituitary and tooth morphogenesis. Nature 401:279282.

Liu C, Liu W, Lu MF, Brown NA, Martin JF. 2001. Regulation of left-right asymmetry by thresholds of Pitx2c activity. Development 128:20392048.

Martin DM, Sheldon S, Gorski JL. 2001. CHARGE association with choanal atresia and inner ear hypoplasia in a child with a de novo chromosome translocation t(2;7)(p14;q21.11). Am J Med Genet 99:115119.

North KN, Wu BL, Cao BN, Whiteman DA, Korf BR. 1995. CHARGE association in a child with de novo inverted duplication (14) $(\mathrm{q} 22 \rightarrow \mathrm{q} 24.3)$. Am J Med Genet 57:610-614.

Pagon RA, Graham JM Jr, Zonana J, Yong SL. 1981. Coloboma, congenital heart disease, and choanal atresia with multiple anomalies: CHARGE association. J Pediatr 99:223-227.

Perveen R, Lloyd IC, Clayton-Smith J, Churchill A, van Heyningen V, Hanson I, Taylor D, McKeown C, Super M, Kerr B, Winter R, Black GC. 2000. Phenotypic variability and asymmetry of Rieger syndrome associated with PITX2 mutations. Invest Ophthalmol Vis Sci 41: 2456-2460

Priston M, Kozlowski K, Gill D, Letwin K, Buys Y, Levin AV, Walter MA, Heon E. 2001. Functional analyses of two newly identified PITX2 mutants reveal a novel molecular mechanism for Axenfeld-Rieger syndrome. Hum Mol Genet 10:1631-1638.

Saadi I, Semina EV, Amendt BA, Harris DJ, Murphy KP, Murray JC, Russo AF. 2001. Identification of a dominant negative homeodomain mutation in Rieger syndrome. J Biol Chem 276:23034-23041.

Sadeghi-Nejad A, Senior B. 1974. Autosomal dominant transmission of isolated growth hormone deficiency in iris-dental dysplasia (Rieger's syndrome). J Pediatr 85:644-648.

Semina EV, Reiter R, Leysens NJ, Alward WL, Small KW, Datson NA, Siegel-Bartelt J, Bierke-Nelson D, Bitoun P, Zabel BU, Carey JC, Murray JC. 1996. Cloning and characterization of a novel bicoid-related homeobox transcription factor gene, RIEG, involved in Rieger syndrome. Nat Genet 14:392-399.

Tellier AL, Lyonnet S, Cormier-Daire V, de Lonlay P, Abadie V, Baumann C, Bonneau D, Labrune P, Lacombe D, Le Merrer M, Nivelon A, Philip N, Briard ML, Munnich A. 1996. Increased paternal age in CHARGE association. Clin Genet 50:548-550.

Tellier AL, Cormier-Daire V, Abadie V, Amiel J, Sigaudy S, Bonnet D, de Lonlay- Debeney P, Morrisseau-Durand MP, Hubert P, Michel JL, Jan D, Dollfus H, Baumann C, Labrune P, Lacombe D, Philip N, LeMerrer M, Briard ML, Munnich A, Lyonnet S. 1998. CHARGE syndrome: report of 47 cases and review. Am J Med Genet 76:402-409.

Tellier AL, Amiel J, Delezoide AL, Audollent S, Auge J, Esnault D, EnchaRazavi F, Munnich A, Lyonnet S, Vekemans M, Attie-Bitach T. 2000 Expression of the PAX2 gene in human embryos and exclusion in the CHARGE syndrome. Am J Med Genet 93:85-88. 